\title{
LOCAL INVERSES OF BOREL HOMOMORPHISMS AND ANALYTIC P-IDEALS
}

\author{
SŁAWOMIR SOLECKI
}

Received 25 July 2004

We present a theorem on the existence of local continuous homomorphic inverses of surjective Borel homomorphisms with countable kernels from Borel groups onto Polish groups. We also associate in a canonical way subgroups of $\mathbb{R}$ with certain analytic P-ideals of subsets of $\mathbb{N}$. These groups, with appropriate topologies, provide examples of Polish, nonlocally compact, totally disconnected groups for which global continuous homomorphic inverses exist in the situation described above. The method of producing these groups generalizes constructions of Stevens and Hjorth and, just as those constructions, yields examples of Polish groups which are totally disconnected and yet are generated by each neighborhood of the identity.

\section{Introduction}

Let $G, H$ be topological groups. Let $U \subseteq G$ be a symmetric open neighborhood of 1 . A function $f: U \rightarrow H$ is called a local homomorphism if $f\left(g_{1} g_{2}\right)=f\left(g_{1}\right) f\left(g_{2}\right)$ for any $g_{1}, g_{2} \in U$ with $g_{1} g_{2} \in U$. Local homomorphisms are of importance in the study of Lie groups $[1,8]$. In the present paper, they will appear in the more general context of Polish groups. (For a definition of Polish groups and other topological notions, see the end of the introduction.) We say that a homomorphism $\pi: H \rightarrow G$ between two topological groups is locally invertible by a local homomorphism if there exist a symmetric neighborhood $U$ of 1 in $G$ and a local homomorphism $f: U \rightarrow H$ such that $\pi(f(g))=g$ for all $g \in U$. We say that $\pi$ is invertible by a homomorphism if $f$ in the preceding sentence can be chosen to be a global homomorphism, that is, with $U=G$.

Invertibility of continuous homomorphisms with discrete kernels is of interest in the study of Lie groups. Recently the problem of finding homomorphic inverses for Borel homomorphisms with countable (not necessarily discrete) kernels between more general groups came up in the work of Christensen et al. [2]. In this paper, the authors investigated linearly ordered groups. The proof of their main theorem [2, Theorem 2] reduces to showing that each surjective Borel homomorphism with countable kernel from an Abelian ordered metric Borel group onto $\mathbb{R}$ is invertible by a continuous homomorphism. This was established by them in [2, Theorem 4]. (Instead of metric Borel groups, 
they work with a slightly wider notion of standard Borel group, see [5, Theorem 8.4].) The proof of this result uses particular properties of ordered commutative groups and particular properties of $\mathbb{R}$. In Section 2, we present a general result on inverting Borel homomorphisms with countable kernels from metric Borel groups onto Polish groups. One can think of this result as somewhat analogous in the group context to the Lusin-Novikov theorem [7, Theorem 18.10] on finding Borel selectors for vertical sections of Borel subsets with countable such sections in products of Polish spaces. In fact, the Lusin-Novikov theorem is used in the proof in a crucial way.

Our theorem yields a local homomorphic inverse and, in general, one cannot hope to obtain a global homomorphic inverse. We investigate the question of when global inverses can be found. It is easily proved that it is so if $G$ is a connected, simply connected Lie group. (By setting $G=\mathbb{R}$, here we recover the Christensen-Kanovei-Reeken result [2, Theorem 4] for metric Borel groups.) In Section 3, our aim is to find groups with this property from the opposite end of the spectrum of Polish groups. Polish groups produced there are nonlocally compact and totally disconnected. Our construction generalizes examples due to Stevens [10] and Hjorth [6]. The construction is accomplished by associating in a canonical way a Polish group $G(I)$ with certain analytic P-ideals $I$ of subsets of $\mathbb{N}$.

Other aspects of the structure of the groups $G(I)$, related to their disconnectedness, are of interest. By a classical result, each totally disconnected, locally compact, second countable group has a neighborhood basis at 1 consisting of closed-and-open subgroups, in particular, such groups are not generated by small enough neighborhoods of 1 [ 8 , Theorem 17], [3, Theorem 6.2.9]. The example produced by Stevens in [10], in answer to a question from the Scottish Book, was the first group which was Polish and both totally disconnected and generated by any neighborhood of 1 . Another example of this sort was constructed by Hjorth [6]. These examples differ in their degree of disconnectedness: Stevens' group is positive dimensional and Hjorth's is zero dimensional. In Section 4, we point out that the groups $G(I)$ are Polish totally disconnected groups with each neighborhood of 1 generating $G(I)$ and that by varying $I$ we can obtain both zero-dimensional and positive-dimensional examples. Moreover, we give a general sufficient condition on $I$ for $G(I)$ to be positive dimensional: there exists an ideal $J$ such that $J$ is below $I$ in the Rudin-Blass ordering and $J$ is an $F_{\sigma}$ P-ideal which is not a trivial modification of the ideal of finite subsets of $\mathbb{N}$.

A topological space is called Polish if it is metric complete separable. A metric separable space is analytic if it is the continuous image of a Polish space. All Borel subsets of Polish spaces are analytic. A set is called $F_{\sigma}$ if it is a countable union of closed sets. So all $F_{\sigma}$ sets are Borel. All groups in the paper will be assumed to be topological groups. By a Polish group, we understand a topological group with a Polish group topology. A group is metric Borel if it is a metric separable group which is Borel in some (or equivalently all) of its metric completions. Of course, all Polish groups are metric Borel.

If $X$ is a metric separable space, by $\operatorname{dim}(X)$, we denote the topological dimension of $X$. Since we will be applying the notion of dimension to metric separable spaces only, by [3, Theorem 7.3.3], it makes no difference which notion of dimension from [3] we choose. In particular, a metric separable space is zero dimensional if it has a topological basis 
consisting of closed-and-open sets. Recall that a metric separable space is totally disconnected if for any two distinct points $x$ and $y$ there exists a closed-and-open set containing $x$ and not containing $y$.

By $\mathbb{N}$, we denote the set of natural numbers including 0 . Let $\mathbb{Q}_{2}$ stand for the group of diadic rationals, that is, all rational numbers whose denominators are powers of 2 with addition as the group operation.

\section{Local inverses}

THEOREM 2.1. Let $\pi$ be a surjective Borel homomorphism from a Borel metric group onto a Polish group. If $\pi$ has countable kernel, then it is locally invertible by a continuous local homomorphism.

Proof. If $U$ is an open subset of a Polish space, we write $\forall^{*} x \in U \ldots$ for "the set $\{x \in U$ : ... is comeager in $U$."

Let $B$ be a metric Borel group, $G$ a Polish group, and $\pi: B \rightarrow G$ a surjective Borel homomorphism with countable kernel. By the uniformization theorem for Borel sets with countable sections [7, Theorem 18.10], there exists a Borel function $f: G \rightarrow B$ such that $\pi \circ f=\mathrm{id}_{G}$. Put $K=\operatorname{ker}(\pi)$. Note that since $\pi$ is a homomorphism, for any $x, y, z \in G$,

$$
\pi\left(f\left(x y^{-1} z\right)^{-1} f(x) f(y)^{-1} f(z)\right)=\left(x y^{-1} z\right)^{-1} x y^{-1} z=1
$$

so $f\left(x y^{-1} z\right)^{-1} f(x) f(y)^{-1} f(z) \in K$. Therefore, since $K$ is countable, we can find $U, V, W$ $\subseteq G$ nonempty open and $g_{0} \in K$ such that

$$
\forall^{*}(x, y, z) \in U \times V \times W, \quad f(x) f(y)^{-1} f(z)=f\left(x y^{-1} z\right) g_{0} .
$$

By making $U$ smaller if necessary, we can make sure that there exists $x_{0} \in G$ so that $x_{0} V \supseteq$ $U$. Since $\pi$ is onto, we can pick $b_{0} \in B$ with $\pi\left(b_{0}\right)=x_{0}$. Note now that

$$
f(y) f\left(x_{0}^{-1} y\right)^{-1} b_{0}^{-1} \in K \quad \text { for any } y \in G \text {. }
$$

Thus, there exist $g_{1} \in K$ and $U_{1} \subseteq U$ nonempty open such that

$$
\forall^{*} y \in U_{1}, \quad f(y)=g_{1} b_{0} f\left(x_{0}^{-1} y\right)
$$

Note also that $x_{0}^{-1} U_{1} \subseteq V$. We can make $U_{1}$ smaller, if necessary, to guarantee that for some $x_{1}, W x_{1} \supseteq x_{0}^{-1} U_{1}$. Pick now $b_{1} \in B$ with $\pi\left(b_{1}\right)=x_{1}$. Then we see that

$$
b_{1}^{-1} f\left(x_{0}^{-1} z x_{1}^{-1}\right)^{-1} b_{0}^{-1} g_{1}^{-1} f(z) \in K
$$

by applying $\pi$ to the left-hand side. It follows from it that for some nonempty open set $U_{2} \subseteq U_{1}$ and $g_{2} \in K$ we have

$$
\forall^{*} z \in U_{2}, \quad f(z)=g_{1} b_{0} f\left(x_{0}^{-1} z x_{1}^{-1}\right) b_{1} g_{2} .
$$


Note that $x_{0}^{-1} U_{2} \subseteq V$ and $x_{0}^{-1} U_{2} x_{1}^{-1} \subseteq W$. We also keep in mind that the KuratowskiUlam theorem [7, Theorem 8.41] gives that $\forall^{*}(x, y, z) \in U_{2}^{3}$ and $\forall^{*} x \in U_{2} \forall^{*} y \in U_{2}$ $\forall^{*} z \in U_{2}$ are equivalent and that multiplications by $x_{0}^{-1}$ and by $x_{1}^{-1}$ are homeomorphisms. Thus, by (2.4) and (2.6), we get the first equality below and by (2.2) the third one:

$$
\begin{aligned}
\forall^{*}(x, y, z) \in U_{2}^{3}, \quad f(x) f(y)^{-1} f(z) & =f(x)\left(f\left(x_{0}^{-1} y\right)^{-1} b_{0}^{-1} g_{1}^{-1}\right)\left(g_{1} b_{0} f\left(x_{0}^{-1} z x_{1}^{-1}\right) b_{1} g_{2}\right) \\
& =f(x) f\left(x_{0}^{-1} y\right)^{-1} f\left(x_{0}^{-1} z x_{1}^{-1}\right) b_{1} g_{2} \\
& =f\left(x\left(x_{0}^{-1} y\right)^{-1}\left(x_{0}^{-1} z x_{1}^{-1}\right)\right) g_{0} b_{1} g_{2} \\
& =f\left(x y^{-1} z x_{1}^{-1}\right) g_{0} b_{1} g_{2} .
\end{aligned}
$$

It follows that there exists a comeager in $U_{2} \times U_{2} \times U_{2}$ set $A \subseteq U_{2} \times U_{2} \times U_{2}$ such that

$$
\forall(x, y, z),(\bar{x}, \bar{y}, \bar{z}) \in A, \quad \text { if } x y^{-1} z=\bar{x} \bar{y}^{-1} \bar{z}, \quad \text { then } f(x) f(y)^{-1} f(z)=f(\bar{x}) f(\bar{y})^{-1} f(\bar{z}) \text {. }
$$

Let $D \subseteq G$ be comeager and such that $f \uparrow D$ is continuous [7, Theorem 8.38]. We claim that for all $x, y, z, \bar{x}, \bar{y}, \bar{z} \in D \cap U_{2}$, we have

$$
\text { if } x y^{-1} z=\bar{x} \bar{y}^{-1} \bar{z}, \quad \text { then } f(x) f(y)^{-1} f(z)=f(\bar{x}) f(\bar{y})^{-1} f(\bar{z}) .
$$

To see it fix $x, y, z, \bar{x}, \bar{y}, \bar{z} \in D \cap U_{2}$. Let $O_{1}$ and $O_{2}$ be open sets with $(x, y, z) \in O_{1} \subseteq U_{2}^{3}$ and $(\bar{x}, \bar{y}, \bar{z}) \in O_{2} \subseteq U_{2}^{3}$. Since $D^{3}$ is comeager in $U_{2}^{3}$ (this is Kuratowski-Ulam again), $D^{3} \cap$ $A \cap O_{1}$ and $D^{3} \cap A \cap O_{2}$ are comeager in $O_{1}$ and $O_{2}$, respectively. Since $G^{3} \ni(a, b, c) \rightarrow$ $a b^{-1} c \in G$ is continuous and open, both

$$
\begin{aligned}
& \left\{x_{1} y_{1}^{-1} z_{1}:\left(x_{1}, y_{1}, z_{1}\right) \in D^{3} \cap A \cap O_{1}\right\} \\
& \left\{x_{1} y_{1}^{-1} z_{1}:\left(x_{1}, y_{1}, z_{1}\right) \in D^{3} \cap A \cap O_{2}\right\}
\end{aligned}
$$

are comeager in some neighborhood of $x y^{-1} z=\bar{x} \bar{y}^{-1} \bar{z}$. Thus, we can find triples $\left(x_{1}, y_{1}, z_{1}\right)$ $\in D^{3} \cap A \cap O_{1}$ and $\left(\bar{x}_{1}, \bar{y}_{1}, \bar{z}_{1}\right) \in D^{3} \cap A \cap O_{2}$ with $x_{1} y_{1}^{-1} z_{1}=\bar{x}_{1} \bar{y}_{1}^{-1} \bar{z}_{1}$. Using this observation, we produce two sequences $\left(x_{n}, y_{n}, z_{n}\right)$ and $\left(\bar{x}_{n}, \bar{y}_{n}, \bar{z}_{n}\right)$ so that $x_{n} \rightarrow x, y_{n} \rightarrow y$, $z_{n} \rightarrow z, \bar{x}_{n} \rightarrow \bar{x}, \bar{y}_{n} \rightarrow \bar{y}, \bar{z}_{m} \rightarrow \bar{z}, x_{n}, y_{n}, z_{n}, \bar{x}_{n}, \bar{y}_{n}, \bar{z}_{n} \in D,\left(x_{n}, y_{n}, z_{n}\right),\left(\bar{x}_{n}, \bar{y}_{n}, \bar{z}_{n}\right) \in A$, and $x_{n} y_{n}^{-1} z_{n}=\bar{x}_{n} \bar{y}_{n}^{-1} \bar{z}_{n}$. It follows by $(2.8)$ that

$$
\begin{aligned}
f(x) f(y)^{-1} f(z) & =\lim _{n} f\left(x_{n}\right) f\left(y_{n}\right)^{-1} f\left(z_{n}\right) \\
& =\lim _{n} f\left(\bar{x}_{n}\right) f\left(\bar{y}_{n}\right)^{-1} f\left(\bar{z}_{n}\right) \\
& =f(\bar{x}) f(\bar{y})^{-1} f(\bar{z})
\end{aligned}
$$

as we claimed in (2.9). 
Thus, to summarize, we have an open set $U_{2}$ and a comeager in $U_{2}$ set $C \subseteq U_{2}$ such that

$\forall x, y, z, \bar{x}, \bar{y}, \bar{z} \in C, \quad$ if $x y^{-1} z=\bar{x} \bar{y}^{-1} \bar{z}, \quad$ then $f(x) f(y)^{-1} f(z)=f(\bar{x}) f(\bar{y})^{-1} f(\bar{z})$.

We can assume that $C$ is Borel. It follows from (2.12) by taking $z=\bar{z} \in C$ that

$$
\forall x, y, \bar{x}, \bar{y} \in C, \quad \text { if } x y^{-1}=\bar{x} \bar{y}^{-1}, \quad \text { then } f(x) f(y)^{-1}=f(\bar{x}) f(\bar{y})^{-1} .
$$

Now by Pettis' theorem [7, Theorem 9.9], $C C^{-1}$ contains an open neighborhood of 1. Let $U$ be the interior of $C C^{-1}$. Then $U$ is symmetric open and $1 \in U$. Define $h: U \rightarrow B$ by letting

$$
h\left(x y^{-1}\right)=f(x) f(y)^{-1} \quad \text { for } x, y \in C \text { with } x y^{-1} \in U .
$$

Note that $h$ is well defined by (2.13). We need to check that $h\left(z_{1} z_{2}\right)=h\left(z_{1}\right) h\left(z_{2}\right)$ for any $z_{1}, z_{2} \in U$ with $z_{1} z_{2} \in U$. Let $x_{1}, y_{1}, x_{2}, y_{2}, x_{3}, y_{3} \in C$ be such that $z_{1}=x_{1} y_{1}^{-1}, z_{2}=$ $x_{2} y_{2}^{-1}, z_{1} z_{2}=x_{3} y_{3}^{-1}$. Then $x_{1} y_{1}^{-1} x_{2} y_{2}^{-1}=x_{3} y_{3}^{-1}$, whence $x_{1} y_{1}^{-1} x_{2}=x_{3} y_{3}^{-1} y_{2}$. By (2.12), $f\left(x_{1}\right) f\left(y_{1}\right)^{-1} f\left(x_{2}\right)=f\left(x_{3}\right) f\left(y_{3}\right)^{-1} f\left(y_{2}\right)$, hence

$$
\left(f\left(x_{1}\right) f\left(y_{1}\right)^{-1}\right)\left(f\left(x_{2}\right) f\left(y_{2}\right)^{-1}\right)=f\left(x_{3}\right) f\left(y_{3}\right)^{-1},
$$

that is, $h\left(z_{1}\right) h\left(z_{2}\right)=h\left(z_{1} z_{2}\right)$.

The local homomorphism $h$ is clearly Borel since its graph is analytic [7, Theorem 14.12], as for $w \in U,(w, v)$ is in the graph of $h$ precisely when

$$
\exists x, y \in C, \quad x y^{-1}=w, \quad f(x) f(y)^{-1}=v
$$

and this condition is analytic since $C$ and $f$ are Borel. We claim that any Borel local homomorphism is necessarily continuous. Continuity of $h$ at 1 follows by modifying the proof of continuity of Borel homomorphisms [7, Theorem 9.10] into the following argument. Fix an open neighborhood $V_{0}$ of 1 in $B$. Let $V$ be an open neighborhood of 1 in $B$ with $V^{-1} V \subseteq V_{0}$. Let $W$ be an open neighborhood of 1 with $W^{-1} W \subseteq U$. Let $x_{n}$, $n \in \mathbb{N}$, be a sequence of elements of $W$ such that $\left\{h\left(x_{n}\right): n \in \mathbb{N}\right\}$ is dense in $h(W)$. Then $h(W) \subseteq \bigcup_{n} h\left(x_{n}\right) V$. Therefore, for some $n_{0}$, which we fix,

$$
h^{-1}\left(h\left(x_{n_{0}}\right) V\right) \cap W \text { is nonmeager. }
$$

Note that this set is included in $x_{n_{0}} h^{-1}(V)$. Indeed, for $x \in W$ with $h(x) \in h\left(x_{n_{0}}\right) V$, we have that $h\left(x_{n_{0}}\right)^{-1} h(x)$ is in $V$ and $x_{n_{0}}^{-1} x$ is in $U$ since both $x_{n_{0}}$ and $x$ are in $W$. It follows that $h\left(x_{n_{0}}^{-1} x\right) \in V$, that is, $x \in x_{n_{0}} h^{-1}(V)$. Taking this and (2.17) into account, by Pettis' theorem [7, Theorem 9.9], 1 is in the interior of

$$
\left(x_{n_{0}} h^{-1}(V)\right)^{-1} x_{n_{0}} h^{-1}(V)=\left(h^{-1}(V)\right)^{-1} h^{-1}(V) .
$$

Since all points in $\left(h^{-1}(V)\right)^{-1} h^{-1}(V) \cap U$ are mapped by $h$ to $V^{-1} V \subseteq V_{0}$, we see that $1 \in G$ lies in the interior of $h^{-1}\left(V_{0}\right)$, that is, $h$ is continuous at 1 . This immediately implies 
that $h$ is continuous at each point of $U$ since if $x \in U$ and $x_{n} \in U, n \in \mathbb{N}$, converge to $x$, then the sequence $\left(x_{n}^{-1} x\right)$ converges to 1 and is eventually in $U$. Thus, for large enough $n$,

$$
h\left(x_{n}\right)^{-1} h(x)=h\left(x_{n}^{-1} x\right) \longrightarrow 1 \quad \text { as } n \longrightarrow \infty .
$$

As is shown by the obvious example of the homomorphism $\mathbb{T} \ni z \rightarrow z^{2} \in \mathbb{T}$, where $\mathbb{T}$ is $\{z \in \mathbb{C}:|z|=1\}$ taken with multiplication, it is not possible to guarantee the existence of a global inverse which is a homomorphism in the theorem above. We address now the issue of finding global inverses. In the corollary below, we formulate a general condition on $G$ under which the existence of a global inverse can be deduced from the existence of a local one.

Corollary 2.2. Let $G$ be a Polish group with a dense subgroup D. Assume that each nonempty open subset of $D$ generates $D$ and that each continuous local homomorphism of $D$ into a metric Borel group extends to a homomorphism defined on D.

Then each surjective Borel homomorphism with countable kernel from a Borel metric group onto $G$ is invertible by a continuous homomorphism.

The proof of Corollary 2.2 will be given after we show the following straightforward lemma.

Lemma 2.3. Let $G$ be a Polish group with a dense subgroup D such that each nonempty open subset of $D$ generates $D$. Then each nonempty open subset of $G$ generates $G$.

Proof. If $U$ is a nonempty open subset of $G$, then, by density of $D, U \cap D$ is a nonempty open subset of $D$. Thus it generates $D$. It follows, again by density of $D$, that $U$ generates a dense open subgroup $H$ of $G$. Since then each coset of $H$ intersects $H$, we have $G=H$.

Proof of Corollary 2.2. Let $B$ be a metric Borel group. We first prove that a continuous local homomorphism $f: U \rightarrow B$ defined on an open symmetric neighborhood $U$ of 1 in $G$ can be extended to $G$. We start with extending $f \uparrow(U \cap D): U \cap D \rightarrow B$ to a homomorphism $g: D \rightarrow B$. Define now $h: G \rightarrow B$ by letting $h(x)=\lim _{n} g\left(d_{n}\right)$ for some sequence $d_{n} \in D$ with $\lim _{n} d_{n}=x$. Note that this limit exists since by density of $D$ there is some $d \in D$ with $d x \in U$. Then for large enough $n, d d_{n} \in U$ and by continuity of $f$ the limit $\lim _{n} f\left(d d_{n}\right)$ exists (and is equal to $f(d x)$ ). Since, for large enough $n$,

$$
f\left(d d_{n}\right)=g\left(d d_{n}\right)=g(d) g\left(d_{n}\right),
$$

we see that $\lim _{n} g\left(d_{n}\right)$ exists. It follows that the function $h$ is well defined. It is a homomorphism directly from its definition. Furthermore, $h \uparrow U$ coincides with $f \uparrow U$ since this latter function is continuous. Note that $h$ is continuous since it is continuous at 1 .

Now let $\pi: B \rightarrow G$ be a Borel homomorphism onto G. By Theorem 2.1, let $f: U \rightarrow B$ be a continuous local homomorphism which is a local inverse of $\pi$. Let $h: G \rightarrow B$ be a continuous homomorphism extending $f$. Since $U$ generates $G$, it is now easy to see that $h$ is an inverse of $\pi$.

The following corollary describes a more concrete situation in which global homomorphic inverses exist. It will be applied in Section 3 to prove Corollary 3.2. Note that in 
the assumption in Corollary 2.4 we do not assume that the topology $\mathbb{Q}_{2}$ inherits from the inclusion $\mathbb{Q}_{2} \subseteq G$ is the standard order topology on $\mathbb{Q}_{2}$.

Corollary 2.4. Let $G$ be a Polish group containing $\mathbb{Q}_{2}$ as a dense subgroup with the sequence $\left(1 / 2^{n}\right)$ tending to the group identity as $n \rightarrow \infty$. Then each surjective Borel homomorphism with countable kernel from a Borel metric group onto $G$ is invertible by a continuous homomorphism.

Proof. This will follow from Corollary 2.2 since $\mathbb{Q}_{2}$ has all the properties required of $D$. Consider $\mathbb{Q}_{2}$ with the topology it gets from $G$. If $U$ is a symmetric neighborhood of 0 , then for large enough $n \in \mathbb{N}$ we have $1 / 2^{n} \in U$. Thus, the group generated by $U$ is $\mathbb{Q}_{2}$. Similarly, if $h$ is a local homomorphism defined on $U$ and $m \in \mathbb{Z}$, we extend it by letting $\tilde{h}\left(m / 2^{n}\right)=h\left(1 / 2^{n}\right) \cdots h\left(1 / 2^{n}\right)$ if $m \geq 0$ and $\tilde{h}\left(m / 2^{n}\right)=h\left(-1 / 2^{n}\right) \cdots h\left(-1 / 2^{n}\right)$ if $m<0$, where both products have $|m|$ factors and where $n$ is large enough so that $1 / 2^{n} \in U$ holds. It is easily checked that $\tilde{h}$ is well defined and that it is a homomorphism.

\section{Subgroups of $\mathbb{R}$ and analytic P-ideals}

In the classical theory of Lie groups, one shows that any connected, simply connected Lie group fulfills the conditions required of $D$ in Corollary 2.2. (In fact, even more relaxed conditions suffice, see [8, Theorems 63 and 15] and [1, Theorem 13.3].) So by Corollary 2.2 with $G=D$, if $G$ is such a Lie group, a global continuous inverse can be found for any Borel surjective homomorphism with countable kernel from a Borel metric group onto $G$. Here we will produce a class of examples of Polish groups $G$ which are far from being locally compact or connected, yet they admit global inverses. We construct such groups by associating a group $G(I)$ with certain analytic P-ideals $I$.

We describe now the construction. Let $\mathbb{R}_{+}$stand for the set of nonnegative reals. For $x \in \mathbb{R}_{+}$, let $\left(x_{n}\right) \in\{0,1\}^{\mathbb{Z}}$ stand for the binary expansion of $x$ where $n$ runs through $\mathbb{Z}$ and, for some $n_{0}, x_{n}=0$ for all $n<n_{0}$ and where the expansion with finitely many digits equal to 1 is chosen if $x$ is a diadic rational. So $x=\sum\left\{2^{-n}: x_{n}=1\right\}$. Define, for $x \in \mathbb{R}_{+}$,

$$
j(x)=\left\{n \in \mathbb{N}: x_{n} \neq x_{n-1}\right\} .
$$

For a family $I$ of subsets of $\mathbb{N}$, define

$$
G(I)=\left\{x \in \mathbb{R}:\left(x \in \mathbb{R}_{+}, j(x) \in I\right) \text { or }\left(x \notin \mathbb{R}_{+}, j(-x) \in I\right)\right\} .
$$

We will show that if $I$ is an invariant, dense, analytic P-ideal, $G(I)$ becomes a group fulfilling the assumptions of Corollary 2.4. I will now explain the notions in the preceding sentence.

A family $I$ of subsets of $\mathbb{N}$ is called an ideal if it is closed under taking finite unions and subsets. Additionally, if $I$ is an ideal, we will assume that $\{n\} \in I$ for each $n \in \mathbb{N}$ and that $\mathbb{N} \notin I$. These two conditions imply that each element of $I$ has infinite complement in $\mathbb{N}$.

We call an ideal of subsets of $\mathbb{N}$ analytic if it is an analytic subset of $\mathscr{P}(\mathbb{N})$ (which in turn is identified via indicator functions with the metric compact space $\{0,1\}^{\mathbb{N}}$ ). An ideal $I$ is called a $P$-ideal if, for any sequence $a_{n} \in I, n \in \mathbb{N}$, of elements of $I$, there exists $a \in I$ such that $a_{n} \backslash a$ is finite for all $n$. A submeasure is a function $\phi: \mathscr{P}(\mathbb{N}) \rightarrow[0, \infty]$ such that 
$\phi(\varnothing)=0, \phi(a) \leq \phi(b)$ if $a \subseteq b$ and $\phi(a \cup b) \leq \phi(a)+\phi(b)$ for $a, b \subseteq \mathbb{N}$. We say that a submeasure $\phi$ is lower semicontinuous if it is lower semicontinuous as a function from $\mathscr{P}(\mathbb{N})=\{0,1\}^{\mathbb{N}}$ taken with the product topology or, equivalently, if $\phi(a)=\sup \{\phi(d): d \subseteq$ $a, d$ finite $\}$ for any $a \subseteq \mathbb{N}$. If $I$ is an analytic P-ideal, then by [9] it follows that there exists a lower semicontinuous submeasure $\phi: \mathscr{P}(\mathbb{N}) \rightarrow[0, \infty]$ such that $I$ is equal to

$$
\operatorname{Exh}(\phi)=\{a \subseteq \mathbb{N}: \phi(a \backslash\{0,1, \ldots, n\}) \longrightarrow 0 \text { as } n \longrightarrow \infty\} .
$$

We can, and will, assume that $\phi(\{n\})>0$ for each $n \in \mathbb{N}$. (If a $\phi$ does not have this property, consider the submeasure $\phi^{\prime}(a)=\phi(a)+\sum_{n \in a} 2^{-n}$. Then $\phi^{\prime}$ fulfills this condition and $\operatorname{Exh}(\phi)=\operatorname{Exh}\left(\phi^{\prime}\right)$.) An ideal $I=\operatorname{Exh}(\phi)$ carries a topology given by the metric $d(a, b)=$ $\phi(a \triangle b)$ where $a \triangle b$ stands for the symmetric difference of $a$ and $b:(a \backslash b) \cup(b \backslash a)$. As should be evident from the parenthetical remark above, a lower semicontinuous submeasure $\phi$ with $I=\operatorname{Exh}(\phi)$ is not unique, however, the topology on $I$ described above does not depend on $\phi$. It is the unique Polish group topology on $I$ stronger than the one inherited from the inclusion $I \subseteq\{0,1\}^{N}$ where $I$ is considered a group with $\triangle$ as the group operation. We call this topology the submeasure topology. (Proofs of the statements in this paragraph can be found in [9].)

For $a \subseteq \mathbb{N}$, let

$$
a-1=\{n \in \mathbb{N}: n+1 \in a\} .
$$

Call an ideal $I$ of subsets of $\mathbb{N}$ invariant if $a \in I$ implies $a-1 \in I$. An ideal $I$ of subsets of $\mathbb{N}$ is called dense if each infinite subset of $\mathbb{N}$ contains an infinite subset from $I$.

As said above, each analytic P-ideal with the submeasure topology is a Polish group in its own right. The group operation is the symmetric difference or, in other words, it is the coordinatewise addition modulo 2 inherited from $\{0,1\}^{\mathbb{N}}=\mathbb{Z}_{2}^{\mathbb{N}}$. In the theorem below, we associate with each invariant dense P-ideal another Polish group with quite different properties.

Theorem 3.1. Let I be an invariant, dense, analytic P-ideal of subsets of $\mathbb{N}$.

(i) $G(I)$ is a subgroup of $\mathbb{R}$ with a unique Polish group topology $\tau$ stronger than the topology inherited from $\mathbb{R}$.

(ii) $G(I)$ contains $\mathbb{Q}_{2}$ as a $\tau$ dense subgroup and $1 / 2^{n} \rightarrow 0$ in $\tau$ as $n \rightarrow \infty$.

(iii) $G(I) \cap[0,1)$ with $\tau$ is homeomorphic to I with the submeasure topology.

The following corollary is now immediate from Theorem 3.1(i),(ii) and Corollary 2.4.

Corollary 3.2. Let I be an invariant, dense, analytic P-ideal of subsets of $\mathbb{N}$. Any Borel homomorphism with countable kernel from a Borel metric group onto $G(I)$ is invertible by a continuous homomorphism.

Define, for $x, y \in \mathbb{R}_{+}, x \ominus y$ to be $\max (x, y)-\min (x, y)$.

The following lemma will cut our task of proving Theorem 3.1 in half. Its verification is straightforward and we leave it to the reader.

Lemma 3.3. Let $H \subseteq \mathbb{R}_{+}$be a semigroup with addition such that for $x, y \in H, x \ominus y \in H$. Assume that $\tau$ is a topology on $H$ stronger than the one inherited from $\mathbb{R}$. Assume further that 
the functions $H \times H \ni(x, y) \rightarrow x+y \in H$ and $H \times H \ni(x, y) \rightarrow x \ominus y \in H$ are continuous where $H$ is taken with $\tau$ and $H \times H$ is taken with $\tau \times \tau$.

Then $H \cup-H$ is a topological group with the topology given by declaring $U$ open if $U \cap H$ and $-(U \backslash H)$ are both $\tau$ open.

Proof of Theorem 3.1. Let $I$ be an invariant analytic P-ideal and let $\phi$ be a lower semicontinuous submeasure with $I=\operatorname{Exh}(\phi)$. In particular, the submeasure topology on $I$ is given by the complete metric $\phi(a \triangle b)$ for $a, b \in I$. We record now two properties of $\phi$.

Claim 1. (i) For any sequence $a_{n}, n \in \mathbb{N}$, of subsets of $\mathbb{N}$, if $\lim _{n} \phi\left(a_{n}\right)=0$, then $\lim _{n} \phi\left(a_{n}-1\right)=0$.

(ii) $\lim _{n} \phi(\{n\})=0$.

Proof of Claim 1. Point (i) follows from invariance of $I$. Indeed, if it failed, we could find a sequence $a_{n} \in I, n \in \mathbb{N}$, such that for some $\delta>0$ and for all $n, \phi\left(a_{n}\right)<2^{-n}$ and $\phi\left(a_{n}-1\right)>$ $\delta$. The first inequality implies that $\bigcup_{n} a_{n} \in \operatorname{Exh}(\phi)=I$. This inequality also implies that each element of $\mathbb{N}$ belongs to at most finitely many sets $a_{n}$ and so to finitely many sets $a_{n}-1$. Thus, from the second inequality, we get that for each $k \in \mathbb{N}$,

$$
\phi\left(\left(\bigcup_{n} a_{n}\right)-1 \backslash\{0, \ldots, k\}\right)=\phi\left(\bigcup_{n}\left(a_{n}-1\right) \backslash\{0, \ldots, k\}\right)>\delta .
$$

It follows that $\left(\bigcup_{n} a_{n}\right)-1 \notin \operatorname{Exh}(\phi)=I$ contradicting the invariance of $I$.

If (ii) failed, we would be able to find a $\delta>0$ and a strictly increasing sequence $\left(n_{k}\right)_{k}$ of natural numbers with $\phi\left(\left\{n_{k}\right\}\right)>\delta$. Then no infinite subset of $\left\{n_{k}: k \in \mathbb{N}\right\}$ is in $\operatorname{Exh}(\phi)=$ $I$ contradicting the density of $I$. This proves Claim 1.

Define

$$
H(I)=\left\{x \in \mathbb{R}_{+}: j(x) \in I\right\} .
$$

Note that $G(I)=H(I) \cup-H(I)$.

Claim 2. For $x, y \in \mathbb{R}_{+}$, we have

$$
j(x+y), j(x \ominus y) \subseteq j(x) \cup j(y) \cup j(x)-1 \cup j(y)-1 .
$$

Proof of Claim 2. It is sufficient to show that, for any $n \in \mathbb{N}$,

$$
n, n+1 \notin j(x) \cup j(y) \Longrightarrow n \notin j(x+y) \cup j(x \ominus y)
$$

which amounts to proving that if $x_{n-1}=x_{n}=x_{n+1}$ and $y_{n-1}=y_{n}=y_{n+1}$, then $(x+y)_{n-1}=$ $(x+y)_{n}$ and $(x \ominus y)_{n-1}=(x \ominus y)_{n}$. This is done by a direct calculation and we leave it to the reader. Some care needs to be exercised when $x+y$ is a diadic rational and the usual way of adding $x$ to $y$ produces the binary expansion with digits equal to 1 from some point on.

Put

$$
\rho(x, y)=\phi(j(x \ominus y))
$$


Obviously,

$$
\rho(x, y)=\rho(y, x), \quad(\rho(x, y)=0 \Longleftrightarrow x=y) .
$$

Claim 3. For any $\epsilon>0$, there exists $\delta>0$ such that, for any $x, y, z \in H(J)$,

$$
\rho(x, y), \rho(y, z)<\delta \Rightarrow \rho(x, z)<\epsilon .
$$

Proof of Claim 3. Note that $x \ominus z$ is equal to one of the following:

$$
(x \ominus y)+(y \ominus z), \quad(x \ominus y) \ominus(y \ominus z) .
$$

It follows now from Claim 2 that $x \ominus z$ is included in

$$
j(x \ominus y) \cup j(y \ominus z) \cup(j(x \ominus y)-1) \cup(j(y \ominus z)-1)
$$

and the conclusion of the claim follows from Claim 1(i).

Define a topology $\tau$ on $H(I)$ by declaring a set $U \subseteq H(I)$ open if for any $x \in U$ there exists $\epsilon>0$ such that for any $y \in H(I)$ with $\rho(x, y)<\epsilon$ we have $y \in U$. It follows from (3.10) and Claim 3 that this is a Hausdorff topology and that each $x \in H(I)$ lies in the interior of $\{y \in H(I): \rho(x, y)<\epsilon\}$ for each $\epsilon>0$. In particular, the sets $\{y \in H(I): \rho(x, y)<$ $1 /(n+1)\}$ with $n \in \mathbb{N}$ form a countable neighborhood basis at $x$ (consisting of not necessarily open sets).

Verification of (i). First note that uniqueness of a topology on $G(I)$ as in (i) follows from [7, Theorem 9.10].

Since $G(I)=H(I) \cup-H(I)$, to produce a topology as in (i) on $G(I)$, we will apply Lemma 3.3. We check that the assumptions of this lemma hold for $H(I)$ and for the topology $\tau$ on $H(I)$. Immediately, from Claim 2 and invariance of $I$, we get that $H(I)$ is a subsemigroup of $\mathbb{R}_{+}$with the property that $x \ominus y \in H(I)$ whenever $x, y \in H(I)$. We check now that the operations of + and $\ominus$ are continuous when $H(I)$ is equipped with the topology $\tau$. Continuity of + follows from Claim $1(\mathrm{i})$ by the fact that $(x+y) \ominus(w+z)$ is equal to one of the following two elements of $H(I)$ :

$$
(x \ominus w)+(y \ominus z), \quad(x \ominus w) \ominus(y \ominus z)
$$

and hence by Claim 2 is included in

$$
j(x \ominus w) \cup j(y \ominus z) \cup(j(x \ominus w)-1) \cup(j(y \ominus z)-1) .
$$

Continuity of $\ominus$ follows by the same argument since $(x \ominus y) \ominus(w \ominus z)$ is also equal to one of the above two elements of $H(I)$.

It remains to check that $\tau$ is Polish. Since the submeasure topology on $I$ is Polish [9], it will follow immediately from (iii) which is verified below.

Verification of (ii). It suffices to check that $\mathbb{Q}_{2} \cap \mathbb{R}_{+}$is contained in $H(I)$, that it is dense there, and that $1 / 2^{n} \rightarrow 0$ in $\tau$ as $n \rightarrow \infty$. The first assertion is clear. The last assertion is simply Claim 1(ii). For the second assertion, it suffices to see that given $x \in H(I)$ and 
$\epsilon>0$ there is $q \in \mathbb{Q}_{2}$ such that $q \leq x$ and $\phi(j(x-q))<\epsilon$. If $\left(x_{n}\right)$ is a binary expansion of $x$, then since $j(x) \in I=\operatorname{Exh}(\phi)$ we get that $\phi\left(j\left(\sum\left\{2^{-n}: x_{n}=1\right.\right.\right.$ and $\left.\left.\left.n \geq m\right\}\right)\right)<\epsilon$ for large enough $m$. Then $q=\sum\left\{2^{-n}: x_{n}=1\right.$ and $\left.n<m\right\}$ does the job.

Verification of (iii). We check that $H(I) \cap[0,1)$ with $\tau$ is homeomorphic to $I$ with the submeasure topology. For $a \subseteq \mathbb{N}$, let $\chi_{a}$ be the indicator function of $a$. For $a \in I$, define $f(a)$ to be the unique element $x$ of $\mathbb{R}_{+}$such that $x_{0}=\chi_{a}(0)$ and $x_{n+1}=x_{n}+\chi_{a}(n+$ 1) $\bmod 2$. Note that since for $a \in I, \mathbb{N} \backslash a$ is infinite, we have $j(f(a))=a$. Thus, $f$ maps $I$ to $H(I) \cap[0,1)$ and is injective as $j$ is its left inverse. It is easy to see that $f$ is onto. We check that $f$ is continuous and open. Since the topology on $I$ is metric and that on $H(I)$ is first countable, we can work with sequences. Let $\left(a_{n}\right)$ be a sequence of elements of $I$ converging in the submeasure topology to $a \in I$, that is, $\phi\left(a_{n} \triangle a\right) \rightarrow 0$. It is then easy to verify that $\rho\left(f\left(a_{n}\right), f(a)\right) \rightarrow 0$. If a sequence $\left(a_{n}\right)$ of elements of $I$ does not have a converging subsequence in the submeasure topology, then since the metric on $I$ induced by $\phi$ is complete, there is $\delta>0$ such that $\phi\left(a_{n} \triangle a_{m}\right)>\delta$ for all $n \neq m$. But then one easily shows that $\inf _{n \neq m} \rho\left(f\left(a_{n}\right), f\left(a_{m}\right)\right)>0$. By Claim 3, we see that $\left(f\left(a_{n}\right)\right)$ does not converge in $H(I)$.

\section{Comments on disconnectedness of the groups $G(I)$}

For definitions of total disconnectedness, zero dimensionality, and dimension, see the introduction. The following corollary shows that the groups $G(I)$ have the properties of Stevens' [10] and Hjorth's [6] groups.

Corollary 4.1. If I is an invariant, dense analytic P-ideal, then $G(I)$ is a Polish totally disconnected group in which each neighborhood of 0 generates the whole group.

Proof. The group $G(I)$ is totally disconnected since, by Theorem 3.1(iii), $G(I) \cap[0,1)$ continuously embeds into a totally disconnected space $\{0,1\}^{\mathbb{N}}$. Each neighborhood of 0 generates the whole group by Lemma 2.3 and Theorem 3.1(ii).

It is not difficult to see that Stevens' group has positive topological dimension. On the other hand, Hjorth's example has dimension 0. By varying the ideal $I$ in our construction and using Theorem 3.1(iii), we can recover both these situations. We will formulate a general result with sufficient conditions for an analytic P-ideal to be positive dimensional.

For ideals $I$ and $J$ of subsets of $\mathbb{N}$, we say that $J$ is Rudin-Blass below $I$, in symbols $J \leq_{R B} I$, if there exists a finite-to-one function $h: \mathbb{N} \rightarrow \mathbb{N}$ such that

$$
a \in J \Longleftrightarrow h^{-1}(a) \in I .
$$

We say that an ideal $I$ is a trivial modification of Fin if for some $a_{0} \subseteq \mathbb{N}$ we have

$$
I=\left\{a \subseteq \mathbb{N}: a \cap a_{0} \text { is finite }\right\} .
$$

Proposition 4.2. Let I be an analytic P-ideal and let J be an $F_{\sigma} P$-ideal which is not a trivial modification of Fin. If $J \leq_{R B} I$, then I with the submeasure topology is not zero dimensional.

Proof. First notice that if $h: \mathbb{N} \rightarrow \mathbb{N}$ witnesses $J \leq_{R B} I$, then the function $f: J \rightarrow I$ given by $f(a)=h^{-1}(a)$ gives a homeomorphic embedding of $J$ with the submeasure topology into 
$I$ with its submeasure topology. Here is one way of seeing it. Notice first that the range of $f$ is closed in the submeasure topology as it is equal to $I \cap\left\{h^{-1}(a): a \subseteq \mathbb{N}\right\}$ and the set $\left\{h^{-1}(a): a \subseteq \mathbb{N}\right\}$ is closed in the product topology on $\{0,1\}^{\mathbb{N}}$ which on $I$ is contained in the submeasure topology. Thus, the range of $f$ when taken with the submeasure topology on $I$ is Polish. Further note that $f$ is an injective homomorphism when we consider $J$ and $I$ to be groups with $\triangle$ as the group operation. Moreover, $\{0,1\}^{\mathbb{N}} \ni a \rightarrow h^{-1}(a) \in\{0,1\}^{\mathbb{N}}$ is continuous in the product topology, therefore, $f$ is a Borel function when $J$ and $I$ are taken with the submeasure topology. (This is because the submeasure topology is Polish and stronger than the product topology, so it has the same Borel sets as the product topology restricted to $I$ and J.) Thus, $f$ is a Borel homomorphism between $J$ and a Polish group which is a closed subgroup of $I$. It follows that $f$ is continuous and open, that is, a homeomorphism.

Thus, if $\operatorname{dim}(J)>0$, then $\operatorname{dim}(I)>0$. We may, therefore, assume that $I$ is equal to an $F_{\sigma}$ P-ideal which is not a trivial modification of Fin. In this situation, by [9], we can find a lower semicontinuous submeasure $\phi$ such that $I=\operatorname{Exh}(\phi)$ and additionally

$$
I=\{a \subseteq \mathbb{N}: \phi(a)<\infty\} .
$$

Let $U$ be an open set in the submeasure topology containing $\varnothing$ and included in $\{a \subseteq$ $\mathbb{N}: \phi(a)<1\}$. We show that its boundary is nonempty. This will prove that $I$ is not zero dimensional. First note that, for each $\epsilon>0,\{n \in \mathbb{N}: \phi(\{n\})<\epsilon\} \notin I$. Otherwise, if we let $a_{0}=\{n: \phi(\{n\}) \geq \epsilon\}$, then

$$
I=\left\{a \subseteq \mathbb{N}: a \cap a_{0} \text { is finite }\right\}
$$

which would make $I$ a trivial modification of Fin. It follows that for any $\epsilon>0$ and any $k$ the set $\{n: n>k$ and $\phi(\{n\})<\epsilon\}$ is not in $I$ and consequently, by (4.3),

$$
\phi(\{n: n>k, \phi(\{n\})<\epsilon\})=\infty .
$$

This equality combined with lower semicontinuity of $\phi$ and the inclusion $U \subseteq\{a \subseteq \mathbb{N}$ : $\phi(a)<1\}$ allows us to pick a sequence $\left(c_{k}\right)$ of finite subsets of $\mathbb{N}$ such that, for each $k$, $\max c_{k}<\min c_{k+1}$,

$$
\bigcup_{i \leq k} c_{i} \in U, \quad \bigcup_{i \leq k} c_{i} \cup\{n\} \notin U \quad \text { for some } n \text { with } \phi(\{n\})<\frac{1}{k+1} .
$$

The first of the conditions in (4.6), lower semicontinuity of $\phi,(4.3)$, and $U \subseteq\{a \subseteq \mathbb{N}$ : $\phi(a)<1\}$ insure that $\bigcup_{k} c_{k}$ is an element of $I$. It follows also that it is in $\bar{U}$. The second condition implies that $\bigcup_{k} c_{k} \in I \backslash U$. So $\bigcup_{k} c_{k}$ lies on the boundary of $U$.

Examples 4.3. We give now two examples of analytic P-ideals: one is one dimensional and the other one is zero dimensional. For many others, the reader may consult [4].

Let $\mathscr{I}_{1 / n}=\left\{a \subseteq \mathbb{N}: \sum_{n \in a} 1 /(n+1)<\infty\right\}$. One readily checks that $\mathscr{I}_{1 / n}$ is an invariant, dense, $F_{\sigma}$ P-ideal. Thus, by Proposition 4.2 and Theorem 3.1(iii), $\operatorname{dim}\left(G\left(\mathscr{I}_{1 / n}\right)\right)>0$. In fact, it is not difficult to check that $\operatorname{dim}\left(\mathscr{I}_{1 / n}\right) \leq 1$, so $G\left(\mathscr{I}_{1 / n}\right)$ is one dimensional. 
Let $\mathscr{L}_{0}=\left\{a \subseteq \mathbb{N}: \lim _{n}(1 /(n+1))|\{i \in a: i \leq n\}|\right\}$. It is immediate that $\mathscr{L}_{0}$ is an invariant dense ideal. It is easy to check that it is an analytic P-ideal, in fact,

$$
\phi(a)=\sup _{n} \frac{1}{n+1}|\{i \in a: i \leq n\}|
$$

is a lower semicontinuous submeasure with $\mathscr{L}_{0}=\operatorname{Exh}(\phi)$. Using this $\phi$ and the very definition of the submeasure topology on $\mathscr{E}_{0}$, one proves without difficulty that $\operatorname{dim}\left(\mathscr{E}_{0}\right)=0$. Thus, by Theorem 3.1(iii), $\operatorname{dim}\left(G\left(\mathscr{L}_{0}\right)\right)=0$.

In the light of Proposition 4.2, the following question seems natural. A positive answer to it would clarify the condition of zero dimensionality for the groups $G(I)$.

Question 4.4. Let $I$ be an analytic P-ideal. Are the following two conditions equivalent?

(a) The ideal $I$ is not zero dimensional with its submeasure topology.

(b) There exists an $F_{\sigma}$ P-ideal $J$ which is not a trivial modification of Fin such that $J \leq_{R B} I$.

\section{Acknowledgment}

This research is supported by NSF Grant DMS-0400931.

\section{References}

[1] D. Bump, Lie Groups, Graduate Texts in Mathematics, vol. 225, Springer, New York, 2004.

[2] J. R. P. Christensen, V. Kanovei, and M. Reeken, On Borel orderable groups, Topology Appl. 109 (2001), no. 3, 285-299.

[3] R. Engelking, General Topology, Monografie Matematyczne, vol. 60, PWN-Polish Scientific Publishers, Warsaw, 1977.

[4] I. Farah, Analytic quotients: theory of liftings for quotients over analytic ideals on the integers, Mem. Amer. Math. Soc. 148 (2000), no. 702, p. xvi+177.

[5] G. Hjorth, Classification and Orbit Equivalence Relations, Mathematical Surveys and Monographs, vol. 75, American Mathematical Society, Rhode Island, 2000.

[6] - A new zero-dimensional Polish group, preprint, 1998.

[7] A. S. Kechris, Classical Descriptive Set Theory, Graduate Texts in Mathematics, vol. 156, Springer, New York, 1995.

[8] L. Pontrjagin, Topological Groups, Princeton Mathematical Series, vol. 2, Princeton University Press, New Jersey, 1939.

[9] S. Solecki, Analytic ideals and their applications, Ann. Pure Appl. Logic 99 (1999), no. 1-3, 5172.

[10] T. C. Stevens, Connectedness of complete metric groups, Colloq. Math. 50 (1986), no. 2, 233240.

Sławomir Solecki: Department of Mathematics, University of Illinois at Urbana-Champaign, 1409 W. Green Street, Urbana, IL 61801, USA

E-mail address: ssolecki@math.uiuc.edu 


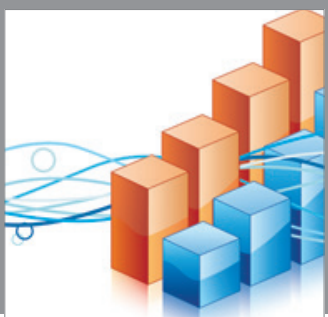

Advances in

Operations Research

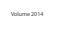

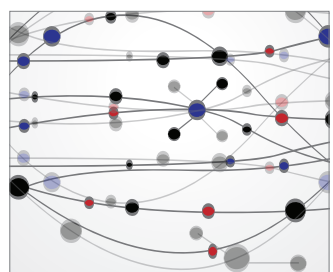

\section{The Scientific} World Journal
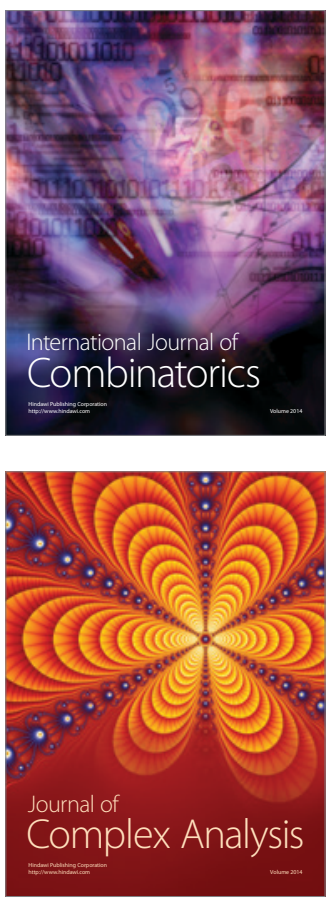

International Journal of

Mathematics and

Mathematical

Sciences
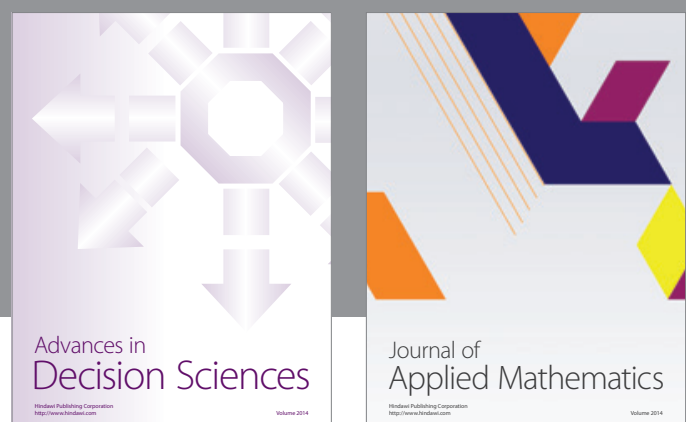

Journal of

Applied Mathematics
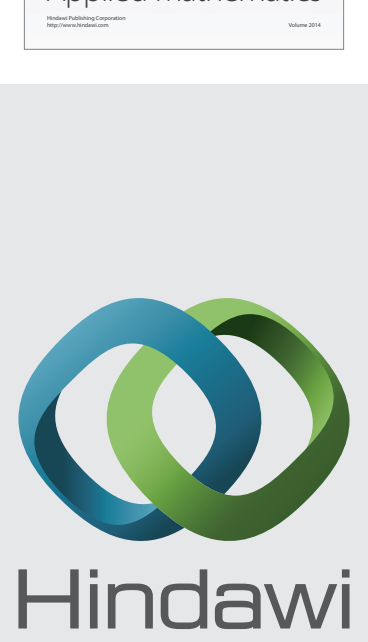

Submit your manuscripts at http://www.hindawi.com
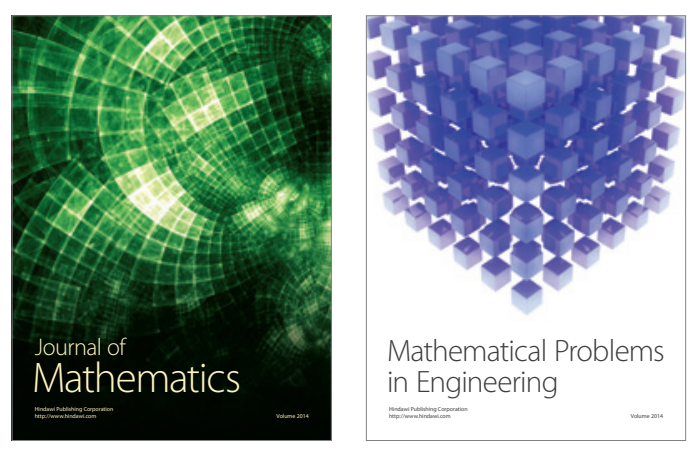

Mathematical Problems in Engineering
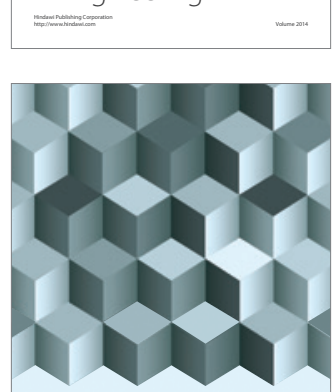

Journal of

Function Spaces
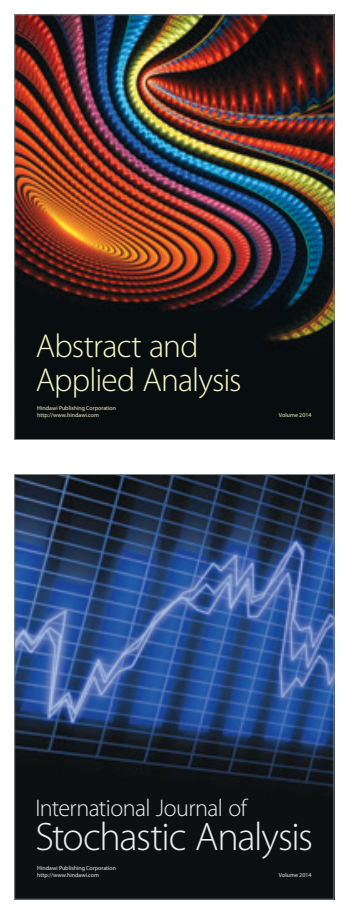

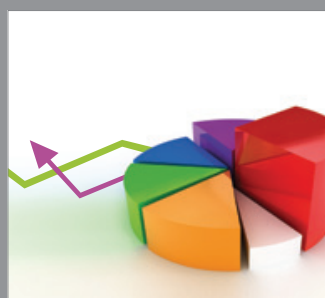

ournal of

Probability and Statistics

Promensencen
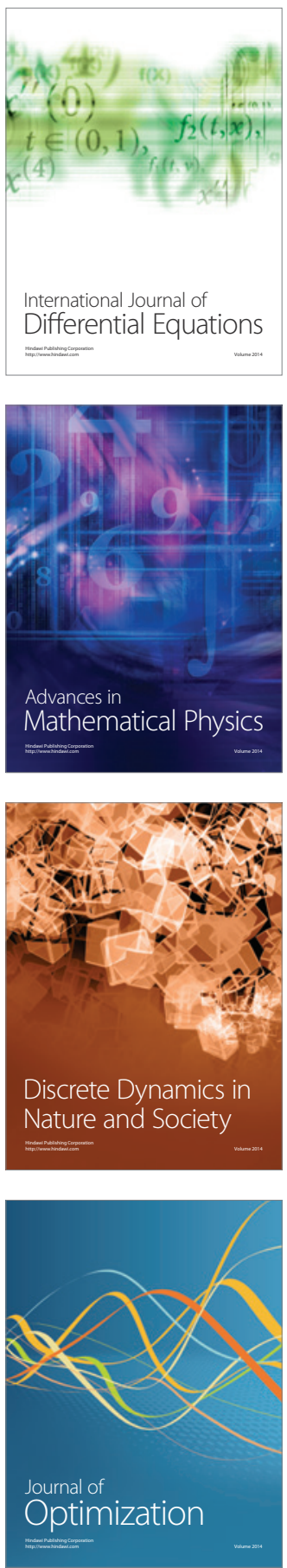